\section{$\underset{\text { hommes }}{\text { \& migrations }}$}

\section{Hommes \& migrations}

Revue française de référence sur les dynamiques

migratoires

\section{$1279 \mid 2009$}

L'Afrique en mouvement

\title{
Ma proche banlieue
}

Entretien avec Patrick Zachmann

\section{Maya Larguet et Patrick Zachmann}

\section{(2) OpenEdition}

\section{Journals}

Édition électronique

URL : http://journals.openedition.org/hommesmigrations/349

DOI : 10.4000/hommesmigrations.349

ISSN : 2262-3353

Éditeur

Musée national de l'histoire de l'immigration

\section{Édition imprimée}

Date de publication : 1 mai 2009

Pagination : 174

ISSN : 1142-852X

\section{Référence électronique}

Maya Larguet et Patrick Zachmann, « Ma proche banlieue », Hommes \& migrations [En ligne], 1279 |

2009, mis en ligne le 29 mai 2013, consulté le 22 septembre 2020. URL : http://

journals.openedition.org/hommesmigrations/349; DOI : https://doi.org/10.4000/hommesmigrations. 349

Ce document a été généré automatiquement le 22 septembre 2020.

Tous droits réservés 


\title{
Ma proche banlieue
}

\author{
Entretien avec Patrick Zachmann \\ Maya Larguet et Patrick Zachmann
}

\section{NOTE DE L'ÉDITEUR}

Patrick Zachmann est photographe. Depuis plus de vingt-cinq ans, il s'intéresse aux questions liées à l'identité, à la mémoire et à l'immigration, que ce soit dans le cadre de ses travaux personnels ou des commandes gérées par l'agence Magnum à laquelle il appartient depuis 1985. La Cité nationale de l'histoire de l'immigration lui consacre une exposition monographique intitulée "Ma proche banlieue".

Comment êtes-vous arrivé à la photo?

Lorsque j'étais enfant, il n'y avait chez nous aucune photo, pas de trace visible de l'histoire. Cela a sûrement joué. Mais j'ai été très tôt attiré par la photographie. Je la pratiquais en amateur averti, puis dans un club où l'on réfléchissait aux conditions de notre pratique. J'ai découvert Cartier-Bresson, Diane Arbus, Brassaï, Robert Frank, Guy Le Querrec avec qui j'ai fait un stage, et d'autres encore qui m'ont influencé. J'en ai fait mon moyen d'expression, bien que mes parents espéraient me voir devenir ingénieur ou architecte. La passion de la photo était devenue quelque chose d'essentiel et de vital. J'ai compris que si j'étais devenu photographe, c'est que j'avais un problème avec la mémoire.

Pouvez-vous nous en dire plus?

Pendant longtemps, j'ai cru que mes parents ne nous avaient rien transmis de la culture juive ni des origines familiales, avant de m'apercevoir que je m'étais trompé. Mais, à l'époque, je ne me sentais ni Juif ni Français. Je ne savais rien de l'histoire de ma famille et j'en souffrais. En fait, ma mère, originaire d'Algérie, et mon père, né à Paris mais originaire de Pologne, gardaient le silence afin d'oublier un passé douloureux. Mon père avait été traumatisé par la guerre : ses parents avaient été déportés après avoir été dénoncés et il a lui-même connu l'holocauste et été détenu en camp de travail. De plus, mes parents étaient immigrés et avaient ce désir très fort 
d'appartenir à la République, d'en respecter les règles, de s'intégrer. La France représentait un réel espoir d'ascension sociale. Mais on ne peut pas se construire si on ne sait pas d'où l'on vient. Et on peut facilement céder à la tentation du communautarisme ou de l'extrémisme.

\section{En quoi la photographie vous a-t-elle aidé?}

Elle m'a permis de mener un travail identitaire et de mettre au jour la mémoire familiale. Les photos sont un miroir du monde et de nous-mêmes car, il y a toujours de nous en l'autre. Au moment de l'arrestation de Pinochet, j'ai fait un travail sur les traces des disparus durant la dictature, qui m'a mené en Argentine, au Rwanda, en Bosnie, puis, enfin, à Auschwitz. J'ai dû faire ces détours par l'histoire des autres pour parvenir à la mienne. Grâce à la photographie, je me suis compris et réconcilié avec moi-même.

Pourquoi êtes-vous attiré par les questions de mémoire, d'identité, d'immigration?

La photo entretient un lien étroit avec l'identité et la mémoire. On parle d'ailleurs de "photo d'identité". Mais les informations brutes et objectives qu'elle donne ne suffisent pas. L'identité suppose de prendre en compte la culture, l'endroit d'où l'on vient, son rapport aux autres, etc. C'est ce qui m'intéresse chez les gens. Mais je ne me considère pas comme un photographe "humaniste". Il y a dans ce mot un côté compassionnel, voire une connotation chrétienne, dans lesquels je ne me reconnais pas. Moi, je suis un photographe d'histoires, un narrateur. Il ne faut pas oublier que je suis fils et petit-fils d'immigrés. Fatalement, l'Ailleurs, même s'il a été occulté, était là. L'autre, le différent, voire l'opposé, m'attirent comme tout ce qui a trait à l'origine et à l'exil. Je suis très influencé par le cinéma et j'ai été bouleversé par America America de Kazan. Ce film a sans doute un peu changé ma vie.

La photographie vous a-t-elle servi de processus analytique?

C'est exact, bien qu'une partie de la profession rejette cet aspect de la photographie. Certains ne voient la photo, ou ne veulent la voir, que comme un acte de journalisme ou une démarche artistique. Moi, j'aime ces allers-retours entre les aspects analytique, ocumentaire et artistique. Ce serait idiot de le nier.

\section{Comment choisissez-vous vos sujets?}

J'ai deux pratiques. La première, que j'apprécie énormément, est professionnelle, quotidienne et alimentaire. Je réponds à des commandes qui transitent par l'agence Magnum et cela débouche parfois sur des travaux propres. Et il y a mes recherches personnelles pour lesquelles je suis guidé par un besoin vital, une urgence. C'est le cas pour "Enquête d'identité" ou pour mon travail sur le Chili. Même si j'ai souffert du non-dit, finalement je travaille dessus. J'aime la pudeur, je suis attiré par ce qui est suggéré ou qu'on peut lire entre les lignes. Par exemple, je suis fasciné par la mafia, qui est une famille avec tous les silences que cela impose, ou par l'Asie, où tout est mystère.

\section{Qu'est-ce qui caractérise votre pratique?}

Le propre des artistes est d'être animé par une nécessité de dire et de comprendre. J'aime parler de ce dont on ne parle pas, aller là où les autres ne vont pas. Être à l'écart, à la périphérie. Ensuite, je fonctionne par allers-retours. Je travaille longtemps sur les sujets. Jusqu'à saturation parfois, avant de les délaisser pour mieux y revenir. J'aime également les allers-retours dans l'espace. Si on veut comprendre 
les immigrés, leur situation et leur comportement en France, il faut se rendre dans leur pays. Aller voir à l'origine est essentiel.

Avez-vous une responsabilité en tant que faiseur d'images?

C'est évident, car on donne à voir le monde et ses réalités. Or on ne peut pas échapper à certains clichés ni ignorer l'impact de certaines images. Certaines fois, je n'ai pas déclenché, car les conséquences étaient trop importantes, en terme de sécurité ou de douleur que cela pouvait ajouter. Je ne prends une photo que si elle est nécessaire. L'acte photographique est agressif, il peut blesser. Cependant, j'essaie de ne pas trop m'autocensurer au moment de la prise de vue et, par la suite, je décide du sort des images.

Vous privilégiez le noir et blanc. Pour quelles raisons?

J'ai commencé alors que la couleur n'était pas disponible, sinon en diapositive que je n'aimais pas. Et puis il y avait une tradition de la photo sociale et humaniste en noir et blanc. Au-delà de ça, le noir et blanc est une transcription du réel. On voit en couleur, mais on photographie en noir et blanc. Cette interprétation est, d'un point de vue créatif, intéressante. Et puis j'ai toujours aimé sa capacité à faire abstraction du superficiel, des détails qui distraient de l'essentiel. Le noir et blanc est un raccourci vers l'émotion, voilà pourquoi il est le meilleur moyen de photographier les gens. Enfin, j'ai toujours aimé les planches contact liées à l'origine au noir et blanc. Elles sont mes journaux intimes, la mémoire de mon travail.

Votre photographie a-t-elle évolué ?

L'exposition "Ma proche banlieue" retrace plus de vingt-cinq ans de recherches. Elle est certes une traversée de territoires, des banlieues, des questions d'immigration, mais aussi une façon de voir ma photographie évoluer. J'ai travaillé en $24 \times 36$, en $6 \times 6$ et en panoramique. J'ai photographié en noir et blanc, et en couleur. Mais surtout, j'ai évolué vers plus de modernisme et la forme a pris de plus en plus d'importance. Ce qui m'importe aujourd'hui, c'est de donner du sens aux choses tout en privilégiant la forme que, par le passé, j’ai parfois sacrifiée.

Est-ce votre sujet qui entraîne la forme ou l'inverse ?

Le sujet me mène à une forme que je juge pertinente. Pour "Maliens, ici et là-bas", j'ai photographié les Maliens d'Evry en couleur, car, à l'époque, on avait l'habitude de voir les immigrés en noir et blanc, dans une vision misérabiliste et compassionnelle. En faisant ressortir, grâce la couleur, mille détails de la vie quotidienne, comme un salon Conforama par exemple, je montrais leur intégration.

Quel rapport entretenez-vous avec les gens que vous photographiez?

Quand je travaille, j'aime être accompagné par quelqu'un qui connaît le terrain et la langue, car j'apprécie de comprendre les règles et les codes. Je me suis aperçu il y a longtemps de l'importance de l'échange. Le chapitre sur les quartiers nord de Marseille est en cela essentiel. C'est à partir de cette expérience fondatrice que je me suis construit une démarche et une éthique. Dans les années quatre-vingt, durant six mois, j'ai enseigné la photographie à des jeunes en difficulté qui, pour la plupart, étaient issus de l'immigration. Moi, je leur apportais une connaissance et eux m'ont permis de pénétrer leur univers et de les photographier. J'ai compris que pour arriver à de bonnes photos, il faut une qualité d'échange, une générosité réciproque. Cela ne peut jamais passer par l'argent. 
Vous avez réalisé deux documentaires. La photographie ne peut-elle pas tout dire?

La photo est irremplaçable dans sa capacité à saisir les non-dits, à symboliser des situations, à définir une époque. Pour autant, elle a ses limites. Quand elle ne suffit plus, je rajoute de l'écrit, auquel j'ai toujours accordé beaucoup d'importance, du son, ou je passe à la réalisation. Une photographie, une fois prise, c'est déjà du passé. Le film permet de prolonger le récit, il est mouvement, sensation du présent. Mais mes documentaires, sur mon père et les jeunes du quartier nord de Marseille que j'ai essayé de retrouver vingt-trois ans plus tard, sont toujours liés à un travail photographique. Je ne veux pas troquer mon appareil pour un autre medium.

L'exposition que vous consacre la Cité s'intitule "Ma proche banlieue". Vous considérezvous comme un photographe de la banlieue?

Non. Je n'ai jamais directement travaillé sur la banlieue, mais sur des sujets qui m'étaient proches et passaient par elle. La banlieue est une terre de migrants, pas seulement d'étrangers. Dans les jardins ouvriers travaillent beaucoup de gens venus de Sarthe ou de Bretagne, à cause de l'exode rural. Quand j'ai eu envie de regrouper une partie de mon travail sur la France, je me suis aperçu que les travaux sur l'identité et l'immigration passaient souvent par la banlieue, que ce soit le travail sur les Chinois, les Maliens, les jardins ouvriers ou encore ma propre famille. J'ai commencé à imaginer une exposition et un livre autour de ce concept.

Qu'entendez-vous par "Ma proche banlieue" ?

Il y a un jeu de mots avec "proche" et "banlieue", mais cela induit aussi ma vision d'une banlieue que j'ai aimée, même si je suis Parisien. En banlieue, il y a plein d'endroits que je trouve moches, tristes, ennuyeux, mais, en revanche, j'aime la banlieue quand elle est liée aux gens que j'y ai rencontrés. Mais il n'y a pas qu'une banlieue, une réalité, une identité. En fait, c'est une vision plurielle de la banlieue que je veux donner. On a souvent tendance à en dramatiser l'image en montrant les problèmes ou les émeutes. Cela existe, mais il faut aussi montrer la banlieue dans sa banalité et dire que la majorité de la population y vit ou y travaille en toute quiétude, avec une réelle qualité de vie.

La banlieue va-t-elle rester un de vos territoires d'investigation?

J'aimerais passer à autre chose. D'autant que c'est très compliqué aujourd'hui de photographier en banlieue, de travailler en toute indépendance et liberté. On va certes vous laisser y accéder, mais à condition que vous donniez une image positive de certains habitants, d'une association ou d'un caïd. La plupart des magazines publient deux sortes d'images. Soit des photos complaisantes de gens qui posent dans des situations négociées, sans regard critique. Soit des images symboliques, des poubelles, des gens de dos ou dont le visage n'apparaît pas pour des questions de droit à l'image et d'argent. Si vous me demandiez d'aller en banlieue demain, je n'irais pas. Ou peut-être avec une bonne raison et quelqu'un qui me permette d'entrer en contact avec la population. Sinon, c'est suicidaire. Vous risquez de vous faire agresser ou voler vos appareils par des imbéciles qui tiennent les cités et pensent avoir tous les droits sur le territoire. Comment trouver l'équilibre entre continuer à témoigner et ne plus rendre compte de ce qui se passe ? Ce n'est pas simple. 
Vous avez photographié la banlieue pendant près de vingt-cing ans. L'avez-vous vue évoluer?

Quand je travaillais dans les quartiers nord de Marseille, dans les années quatrevingt, il y avait encore chez les jeunes issus de l'immigration, un espoir et une volonté de s'intégrer, de faire partie de cette société et de profiter de l'ascenseur social. Même si l'environnement était pauvre et sale, il y avait de la vie, de la solidarité. Vingt-trois ans plus tard, le constat est rude. La drogue et le sida ont décimé.

Il y a une réelle misère sociale, le chômage a fait voler en éclat des équilibres déjà précaires. Il n'y a plus de cohésion sociale ni d'idéal politique. C'est chacun pour soi. Ceux qui ont réussi - car il existe malgré tout des exemples qui donnent des raisons d'espérer - ont dû quitter la cité pour cela. C'est compréhensible puisque ces cités ont été créées pour être des lieux de transit, non des zones d'habitation.

Cependant, cela n'explique pas tout.

\section{C'est-à-dire ?}

Les problèmes de la banlieue me ramènent aux fondements de la République, à laquelle je suis très attaché. Ils posent question sur les raisons d'une faillite, sur les parts de responsabilité de la société, mais aussi des gens eux-mêmes. Pourquoi certains s'intègrent-ils mieux que d'autres? Je m'interroge sans céder au "politiquement correct" qui voudrait que ce soit uniquement la faute de l'État. Beaucoup de choses reposent aussi sur la famille, l'éducation, le respect et la transmission d'une culture et d'une mémoire. Tout n'est pas noir ou blanc. C'est aussi cette vision manichéenne que je conteste avec mes photos et mes films, qui prouvent que les choses sont plus complexes.

Vos travaux jouent-ils également d'autres rôles?

La photo est un miroir. Lorsque vous êtes englués dans le quotidien ou dans vos problèmes, voir la vie par le biais d'un œil extérieur peut aider à avancer. Pour la banlieue, qui a un vrai problème d'identité car son histoire n'est pas inscrite et son enracinement n'est pas précis, la photo peut être un morceau du puzzle qui peut aider à construire une histoire et une mémoire.

Quel sens cela a-t-il pour vous d'exposer à la Cité nationale de l'histoire de l'immigration?

Je suis ravi que mon travail y soit montré, compte tenu de mon parcours et des thématiques qui me sont chères. C'est un aboutissement, la boucle est bouclée. C'est important que ce lieu existe et qu'il évolue avec le temps, car la mémoire est en mouvement, en perpétuelle construction, et les questions liées à l'immigration sont cruciales.

Quel regard portez-vous sur la façon dont ces questions sont gérées en France?

La problématique est sensible, mais essentielle à prendre en compte pour l'avenir de la France. Ce pays s'est en partie construit grâce aux immigrés, et il est normal que ces derniers, ainsi que leurs enfants, soient intégrés, tout comme les nouveaux arrivants. En ce sens, un ministère de l'Immigration est une bonne chose. Cependant, la répression ne peut pas être la seule solution et il faut veiller à ne pas forcément associer "immigration" et "problème". Il faudrait que les politiques puissent débattre sans tabou de ces questions avec l'aide de sociologues, d'historiens et de tous les spécialistes concernés. 
Avez-vous en tête de nouveaux projets ?

Je n'ai plus vraiment envie de travailler en France, car le contrat de confiance est rompu à cause des problèmes liés au droit à l'image, comme je le disais. Pourtant, j'ai envie de travailler sur la nouvelle immigration de l'Est chez nous ainsi que sur les Africains qui arrivent dans des embarcations improbables en Europe.

Un peu obsessionnel, non?

Tout à fait! D'ailleurs, c'est un mot important pour moi. Même si, en apparence, je change de thèmes, de lieux, mon travail reste obsessionnel. C'est ce qui est intéressant justement dans l'œuvre d'un artiste: il ne fait que se répéter, tourner autour des mêmes interrogations, tout en essayant d'en renouveler l'approche créative. Se remettre en question, voilà l'essentiel.

\section{AUTEURS}

\section{PATRICK ZACHMANN}

Photographe 